\title{
ANTICANCER DRUGS AS PROSPECTIVE EFFLUX PUMP INHIBITORS FOR SALMONELLA TYPHI PRODUCE CONFLICTING RESULTS IN IN SILICO AND IN VITRO STUDIES
}

\author{
PRANJALI GUPTA, PANKAJ GAUTAM*, NISHANT RAI
}

Department of Biotechnology, Graphic Era University, Dehradun (Uttarakhand, India) 566/6, Bell Road, Clement Town, Dehradun, Uttarakhand 248002

Email: gautampankaj76@gmail.com

Received: 10 Sep 2016 Revised and Accepted: 18 Oct 2016

\begin{abstract}
Objective: Anticancer drugs paclitaxel and vinblastine were tested for their potential as efflux pump inhibitors for Salmonella Typhi-based on in silico and in vitro studies.

Methods: Three-dimensional protein models of AcrAB-TolC of Salmonella Typhi were generated by online server PHYRE-2. The quality of 3D structures was assessed by PROCHECK, SWISS MODEL. Docking analysis of anticancer drugs with AcrA, AcrB and TolC subunits were performed after refining the homology models with Modrefiner. Salmonella Typhi (S. Typhi) efflux pump activity was measured by ethidium bromide (EtBr) cartwheel and semi-automated fluorometry methods respectively. Fluorescence intensity in bacterial colonies was measured under different treatment conditions (with or without drugs) on Muller Hinton agar (MHA) plates containing EtBr in cartwheel assay. EtBr efflux assay was determined following the loading of bacteria with EtBr and fluorescence was recorded over fixed time period with the help of fluorescent spectrophotometer. The results obtained were compared with the control.
\end{abstract}

Results: Efflux pump inhibitor (EPIs) activity of paclitaxel and vinblastine determined by EtBr cartwheel assay registered no activity whereas semiautomated fluorescent assay revealed marginal activity when compared to control.

Conclusion: We report the conflicting result of in silico and in vitro studies in predicting the antimicrobial effect of mainstream anticancer drugs as efflux pump inhibitors for Salmonella Typhi.

Keywords: Efflux Pump, Efflux pump inhibitor, Salmonella Typhi, Multidrug resistance, AcrAB-TolC, Drug repurposing, Anticancer drugs, Antimicrobial agent

(C) 2016 The Authors. Published by Innovare Academic Sciences Pvt Ltd. This is an open access article under the CC BY license (http://creativecommons.org/licenses/by/4. 0/)

DOI: http://dx.doi.org/10.22159/ijpps.2016v8i12.15136

\section{INTRODUCTION}

Drug resistance in Salmonella species causes significant morbidity and mortality at world level [1] and lack of new antimicrobial agent is further complicating the disease scenario [2]. Incidences of multi-drug resistance (MDR) Salmonella Typhi are growing at an alarming rate and, have been reported from Asia, Europe and the USA [3]. The implication of efflux pumps in MDR Salmonella virulence has been reported by a number of published studies [4]. AcrAB-TolC represents the major category of efflux pump present in Salmonella species and its expression is regulated by RamA in response to external signals [5]. The combination of efflux pump inhibitors (EPIs) with antibiotics has been suggested, among other strategies, for the management of MDRrelated cases in clinical and community settings [6]. Efflux pump inhibitors (EPIs), coming from the natural or synthetic origin, have shown their potential to enhance the antibiotic activity by mechanisms including synergism and potentiation [7]. The large-scale uses of efflux pump inhibitors (EPIs) have been hampered because of the high toxicity observed in experimental studies. Drug repurposing may solve problems related to high toxicity of antimicrobial drugs and offer the advantage of already available pharmacological data for the existing drugs [8]. Anticancer drugs, for example, are reported to have an antibacterial effect in few published reports in in vitro and in silico [9, $10]$, but their mode of action, in the case of pathogenic bacteria, has not been understood in greater depth. Drugs like celecoxib [11] and tirapazamine [12] are known anticancer drugs with defined molecular mechanisms in cancer malignancies, but their antimicrobial effect is poorly understood. Paclitaxel and vinblastine are primarily used for the treatment of human cancers including breast, pancreatic, Hodgkin's lymphoma, lung, and brain cancer. Based on available evidence, we tested selected mainstream anticancer drugs: paclitaxel and vinblastine, for their antimicrobial effect as efflux pump inhibitors against efflux pump of Salmonella Typhi.

\section{MATERIALS AND METHODS}

Chemicals and instrumentation

Bacterial Strain: Resistant MDR (MTCC-3215) and susceptible strains (MTCC-733) of Salmonella Typhi were procured from The Institute of Microbial Technology (IMTECH), Chandigarh, India. Both strains: MTCC-733 and MTCC-3215 were cultured on bismuth sulfite agar (BSA) and tryptic soya agar (TSA) respectively, according to the supplier's protocol. Glycerol stocks of bacterial culture were also stored at $-20{ }^{\circ} \mathrm{C}$ for later use.

Anticancer drugs: Paclitaxel (T1912-1 mg), vinblastine (V1377-1 $\mathrm{mg}$ ) and trimethoprim (TMP) (92131-1G) were obtained from Sigma-Aldrich, USA. Stocks ( $1 \mathrm{mg} / 200 \mu \mathrm{l}$ corresponding to $5 \mathrm{mg} / \mathrm{ml}$ ) of paclitaxel and vinblastine were prepared in one milliliter of dimethyl sulfoxide (DMSO) whereas stock of trimethoprim (25 $\mathrm{mg} / \mathrm{ml}$ ) was prepared in 1:1 molar ratio of chloroform/ethanol.

Antibiotics: Stocks of antibiotics: kanamycin (1 $\mathrm{mg} / \mathrm{ml}$ ) (Cal Biochem, Germany) and ciprofloxacin (1 mg/2 ml) (HiMedia) were prepared in sterile MilliQ water. Two-fold dilutions of paclitaxel and vinblastine (at $160 \mu \mathrm{g} / \mathrm{ml}, 80 \mu \mathrm{g} / \mathrm{ml}, 40 \mu \mathrm{g} / \mathrm{ml}, 20 \mu \mathrm{g} / \mathrm{ml}$, and $10 \mu \mathrm{g} / \mathrm{ml}$ ) were used. The sub-lethal dose of antibiotic (trimethoprim-TMP) was used as mentioned in National Committee for Clinical Laboratory Standards (NCCLS-M02A11, Vol 29, No-1) guidelines [13]. All stock solutions were sterilized by filtration ( $0.22 \mu \mathrm{m}$ filter Merck Millipore) and stored at $2-8{ }^{\circ} \mathrm{C}$ according to the manufacturer instruction (Sigma/HiMedia).

Bioinformatics tools: Pubchem (http://www.ncbi.nlm.nih.gov/ sites/entrez?db=pccompound) Phyre-2 (http://www.sbg.bio.ic.ac.uk/ phyre2/html/page.cgi?id=index), Zinc database (http://zinc. docking.org/), SWISS-MODEL (https://swissmodel.expasy.org/), ModRefiner (http://zhanglab.ccmb.med.umich.edu/ModRefiner/), 
iGEMDOCK software (http://gemdock.life.nctu.edu.tw/dock/ download.php), Autodock Vina (http://vina.scripps.edu/), Cygwin (http://www.cygwin.com/).

Instruments used: UV-gel dock (Genei ${ }^{\mathrm{TM}}$ ), fluorescent spectrophotometer Hitachi (F-4600, Tokyo, Japan).

\section{In silico molecular modeling and docking studies}

Three-dimensional protein models (in pdb format) of each subunit of AcrAB TolC protein complex were generated by the Phyre- 2 online tool (http://www.sbg.bio.ic.ac.uk/phyre2/html/page.cgi?id=index) by using primary sequence (AcrA-CIC61792.1, AcrB-CIC61709.1, and TolC CXA84606.1) from National Centre for Biotechnology Information (NCBI) database [14]. Quality assessment of Phyre-2 generated 3Dmodels was performed by SWISS-MODEL tool. Drug ligands (paclitaxel, vinblastine, and trimethoprim) used in the present study were downloaded from Zinc database (http://zinc.docking.org/) in pdb format [15]. The homology-based three-dimensional model of AcrABTolC were further refined by online tool ModRefiner (http://zhanglab. ccmb.med.umich.edu/ModRefiner/) to resolve the local distortions for higher resolution analysis [16]. Refined models of AcrAB-TolC were used for docking with drug ligands (paclitaxel, vinblastine, and trimethoprim). Autodock Vina was used to set the grid box parameter [17] and iGEMDOCK software was used separately for docking analysis [18]. Graphics of favorable docked poses were visualized by UCSF chimera tool (https://www.cgl.ucsf.edu/chimera/).

\section{Efflux pump overexpression in Salmonella Typhi}

Overexpression of efflux pump was induced (I) by indole according to the previously described protocol [19]. Overnight bacterial suspension was adjusted to $0.3 \mathrm{OD}$ and induced by indole $(4 \mathrm{mmol})$ for $4 \mathrm{~h}$ at $37^{\circ} \mathrm{C}$. Ethidium bromide cartwheel assay was performed according to the previously described method [20] wherein Muller Hinton agar (MHA) plates were mixed with ethidium bromide at $2.5 \mu \mathrm{g} / \mathrm{ml}$ of concentration and indole-induced and uninduced bacteria were swabbed as spokes on the same plates. EtBr plates were kept in the dark and incubated for $24 \mathrm{~h}$ at $37^{\circ} \mathrm{C}$. Next day the differential fluorescence of bacterial colony forming a unit (CFUs) in each spoke was observed in UV-gel dock (fig. 1).

\section{EtBr efflux assays by semi-automated fluorometry method}

\section{Accumulation assay}

Salmonella Typhi was grown at $\pm 35^{\circ} \mathrm{C}$ in Muller Hinton broth (cationsupplemented-MHB M1657 HIMEDIA) till the mid-log phase. Bacterial culture was centrifuged at $10,000 \mathrm{rpm}$ for $10 \mathrm{~min}$ at optical density $\mathrm{OD}_{600} \mathrm{~nm}$ which corresponds to $0.6 \mathrm{DD}$. The bacterial pellet obtained was washed thrice with phosphate buffered saline (1X PBS buffer) solution. The bacterial suspension was then adjusted to the optical density equivalent to 0.30D with PBS followed by addition of EtBr to the final concentration of $6 \mu \mathrm{g} / \mathrm{ml}$ for Salmonella Typhi (MTCC-733). The etbr-loaded bacterial suspension was incubated at $37^{\circ} \mathrm{C}$ for 30 $\min [21]$.

\section{Efflux pump inhibition assay by semi-automated fluorometric assay}

The EtBr-loaded bacterial suspension was centrifuged at 10,000 rpm for $10 \mathrm{~min}$. The pellet was mixed with phosphate buffered saline (PBS) solution and dispensed into different eppendorf tubes for further treatment with drugs. Each aliquot of EtBr loaded bacterial suspension was treated with two-fold dilutions of paclitaxel and vinblastine (ranging from $160 \mu \mathrm{g} / \mathrm{ml}, 80 \mu \mathrm{g} / \mathrm{ml}, 40 \mu \mathrm{g} / \mathrm{ml}, 20 \mu \mathrm{g} / \mathrm{ml}, 10 \mu \mathrm{g} / \mathrm{ml}$ concentration each) and trimethoprim (putative EPI). All experiment was conducted in triplicate. The fluorometric measurement was performed with a fluorescence spectro-photometer (Hitachi fluorescent spectrophotometer F-4600, Tokyo, Japan) (fig. 2). The fluorescence was captured at excitation wavelength $350 \mathrm{~nm}$ and band pass $(10 \mathrm{~nm})$ at the time interval of 45 seconds [22].

\section{RESULTS AND DISCUSSION}

Anticancer drugs produce significant docking with in silico modeled efflux pump proteins of $S$. Typhi; Computational methods have proved highly useful in identification of prospective drug compounds and leading the drug discovery process in the present age. Bioinformatics and drug repurposing are setting a new stage for testing the potential of the known drugs for new indications at relatively low cost and less effort. Drug ligands used in present study bind to all three subunits of AcrAB-TolC efflux complex as evidenced by table 1 , table 2 ; but the binding pockets revealed a different set of interacting amino acids by iGEMDOCK and auto dock vina separately. High-resolution crystallographic structure helped in characterizing the binding pockets in AcrB subunits of E. coli with co-crystallized drugs like doxorubicin and minocycline. These drugs bind to polar (Asparagines-274 and Glutamine-176) (table 3) and hydrophobic phenylalanine (at 136, 178, 610, 615, 617 and 628 positions respectively) in the binding pocket of AcrB [23]. Different drug ligands (paclitaxel and vinblastine) registered variable binding with AcrB of Salmonella Typhi, which may be attributed to the different scoring methods and algorithms employed by the two software tools used in present study. iGEMDOCK employs post-screening analysis docking whereas auto dock vina uses rigid binding sites analysis. Moreover, AcrAB-TolC is a complex efflux protein complex manifesting multiple substrate specificities (ethidium bromide, antibiotics, dyes and the like). The interaction analysis and total free energy obtained for paclitaxel, vinblastine, and trimethoprim (the putative EPI of AcrAB-TolC) revealed an intricate pattern. iGEMDOCK recorded approximately ten times higher total free energy in comparison to Auto dock Vina (table 2 and table 4). A unique observation was made in case of trimethoprim which always produced lesser free energy in comparison to paclitaxel and vinblastine by both iGEMDOCK and Autodock vina.

In present work, the three-dimensional structures for protein subunits: AcrA, AcrB and TolC of Salmonella Typhi, were generated with $100 \%$ confidence value each and with $62 \%, 70 \%$ and $93 \%$ sequence similarity respectively by Phyre-2. High confidence value $(100 \%)$ and significant Ramachandran plot values $(86.7 \%$, 90.5\%, $90.4 \%$ respectively) (fig. 3) together signify the suitability of protein models for subsequent docking analysis (fig. 4). Quality assessment of protein models (AcrA, AcrB and TolC) was performed using PROCHECK, SWISS-MODEL, Autodock Vina online server (fig. 6). A close analysis of Ramachandran values show that most of the amino acids from the modeled structure fall into the favored regions. Grid box parameters including spacing angstrom and grid size for various combinations (subunits of $S$. Typhi efflux AcrAB-TolC paclitaxel, vinblastine and trimethoprim) has been given in (table 5).

Computational docking analysis of in silico protein models with the ligand molecules (trimethoprim, paclitaxel and vinblastine) were undertaken using iGEMDOCK software. The utility of iGEMDOCK is well established ( $78 \%$ success rates) from its testing on 305 proteinligand Astex set [24]. Furthermore, the post-screening analysis and clustering of docked poses based on the high energy scores and pharmacological interactions makes iGEMDOCK more relevant for the present studies (table 1) shows the docking result of trimethoprim, paclitaxel and vinblastine with three subunits (AcrA, AcrB and TolC) of $S$. Typhi. Total free energy scored for paclitaxel was found lowest with all the three subunits: AcrA (-120.71 $\mathrm{kcal} / \mathrm{mol})$, AcrB $(-135.22 \mathrm{kcal} / \mathrm{mol})$ and TolC $(-127.74 \mathrm{kcal} / \mathrm{mol})$ respectively whereas vinblastine recorded only AcrA (-111.8 $\mathrm{kcal} / \mathrm{mol})$, AcrB $(-124.19 \mathrm{kcal} / \mathrm{mol})$ and TolC $(-146.13 \mathrm{kcal} / \mathrm{mol})$ respectively (table 1). Trimethoprim, contrastingly, registered only AcrA $(-89.11 \mathrm{kcal} / \mathrm{mol})$, AcrB $(-84.21 \mathrm{kcal} / \mathrm{mol})$ and TolC $(-90.02$ $\mathrm{kcal} / \mathrm{mol}$ ) respectively. The inclusion of trimethoprim [25], the known inhibitor of AcrAB-TolC efflux pump for Salmonella species facilitates the comparisons of obtained free energies with that of the test drugs (paclitaxel and vinblastine). The Large negative energy of paclitaxel and vinblastine as compared to trimethoprim is largely contributed by the hydrophobic van der Waal's bonds, whereas the contribution of electrostatic energy was entirely missing in overall scored free energies (table 4). AcrAB-TolC being a transmembrane protein seems to have a high propensity for binding with hydrophobic bonds in case of paclitaxel and vinblastine.

The observed trend of the obtained values does not change with the repeat of docking at different time periods. The tabulated total free energy is scored by the sum of van der waal energy ( $\mathrm{vdW}$ ), hydrogen bond energy (H-bond) and electrostatic energy. Each docked pose was considered at RMSD $\leq 2.0 \AA$ 

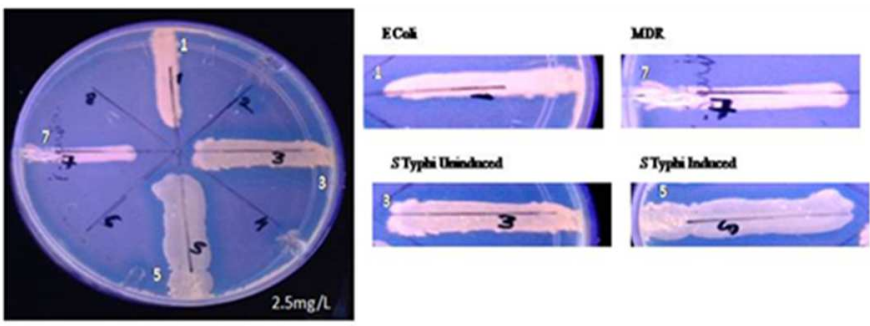

Detection of efllux punpep exprevinen by EBBrartwhed axuy

Fig. 1: Detection of efflux pump expression by EtBr cartwheel assay, MHA plate (containing EtBr at $2.5 \mathrm{mg} / \mathrm{ml}$ ) displaying differential fluorescence by bacteria Spoke 1-Escherichia coli (E. coli) (MTCC-1698), Spoke 3 and 5-Salmonella Typhi (Indole-induced and uninduced MTCC-733) and Spoke 7-Salmonella. Spp MDR-MTCC-3215. Lowest fluorescence was observed in indole-induced $S$. Typhi (Spoke-5) indicates efflux pump expression

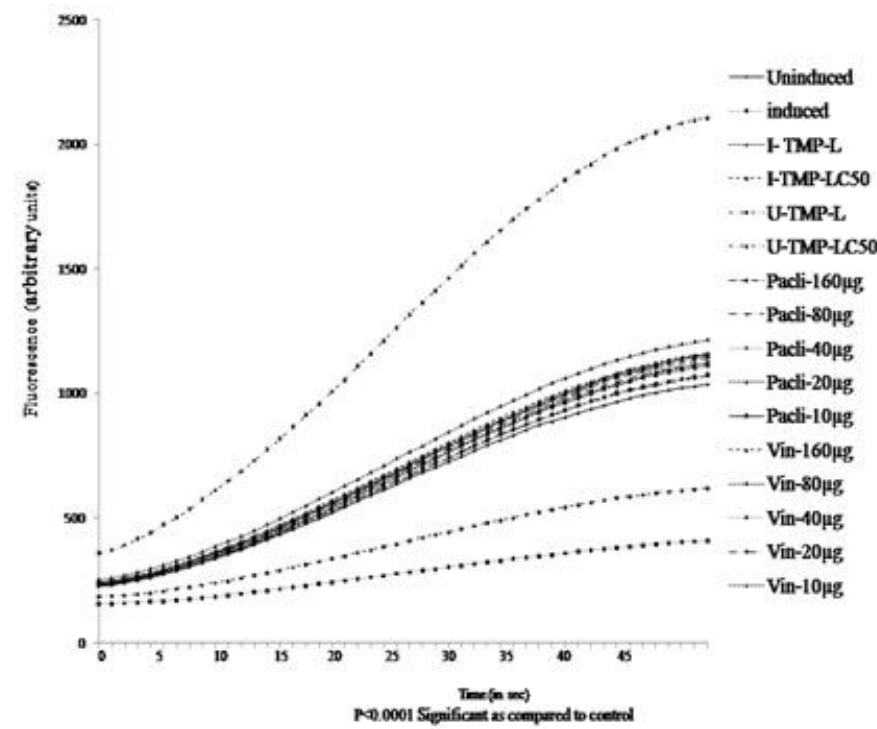

Fig. 2: Graph illustrates the fluorescence produced by uninduced and induced $S$. Typhi after its treatment with trimethoprim (lethal and sub-lethal), paclitaxel $(160 \mu \mathrm{g} / \mathrm{ml}, 80 \mu \mathrm{g} / \mathrm{ml}, 40 \mu \mathrm{g} / \mathrm{ml}, 20 \mu \mathrm{g} / \mathrm{ml}$ and $10 \mu \mathrm{g} / \mathrm{ml}$ respectively) and vinblastine $(160 \mu \mathrm{g} / \mathrm{ml}, 80 \mu \mathrm{g} / \mathrm{ml}$, $40 \mu \mathrm{g} / \mathrm{ml}, 20 \mu \mathrm{g} / \mathrm{ml}$ and $10 \mu \mathrm{g} / \mathrm{ml}$ respectively)
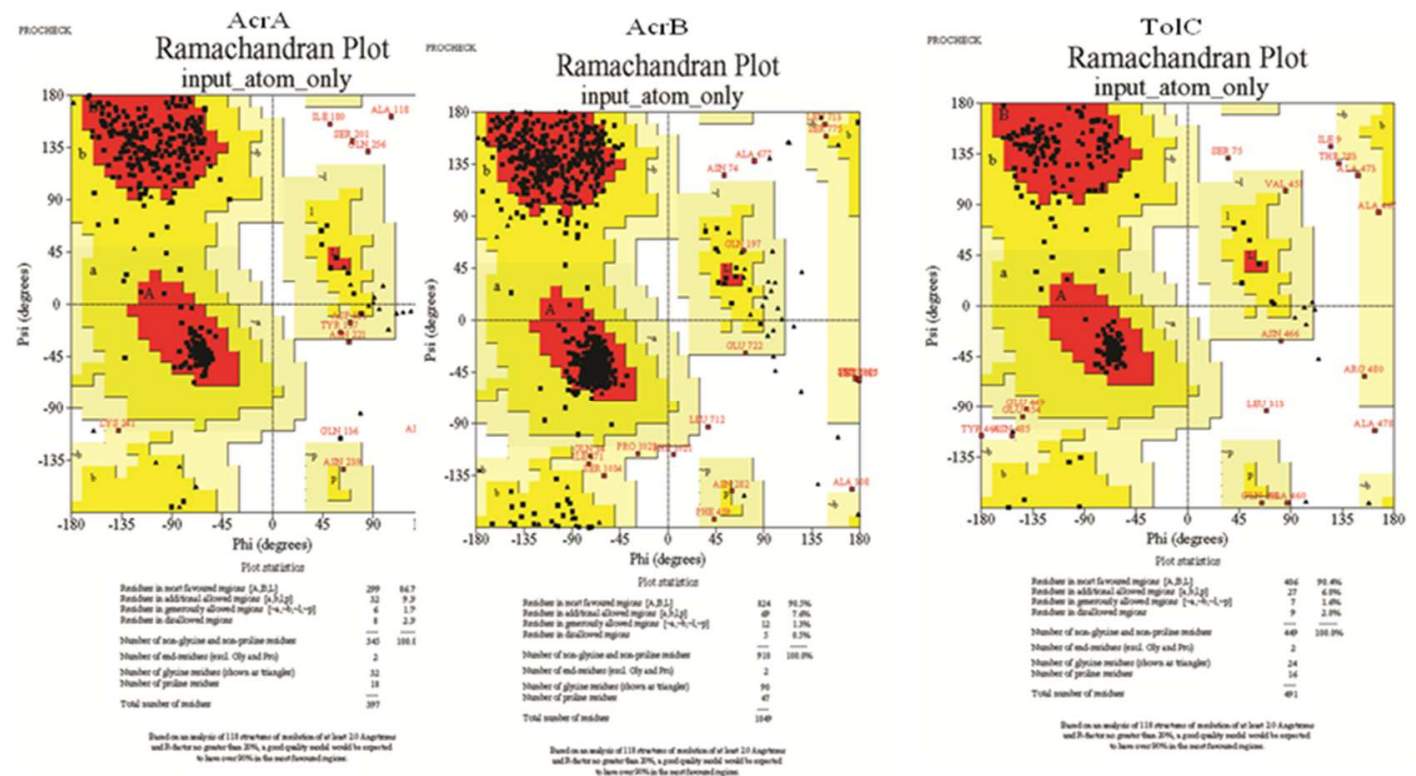

Fig. 3: Ramachandran plots of protein subunits of AcrAB-TolC Salmonella Typhi, AcrA (86.7\%), AcrB (90.5\%), TolC (90.4\%). Black dots representing the amino acids and red region representing the favorable zone 

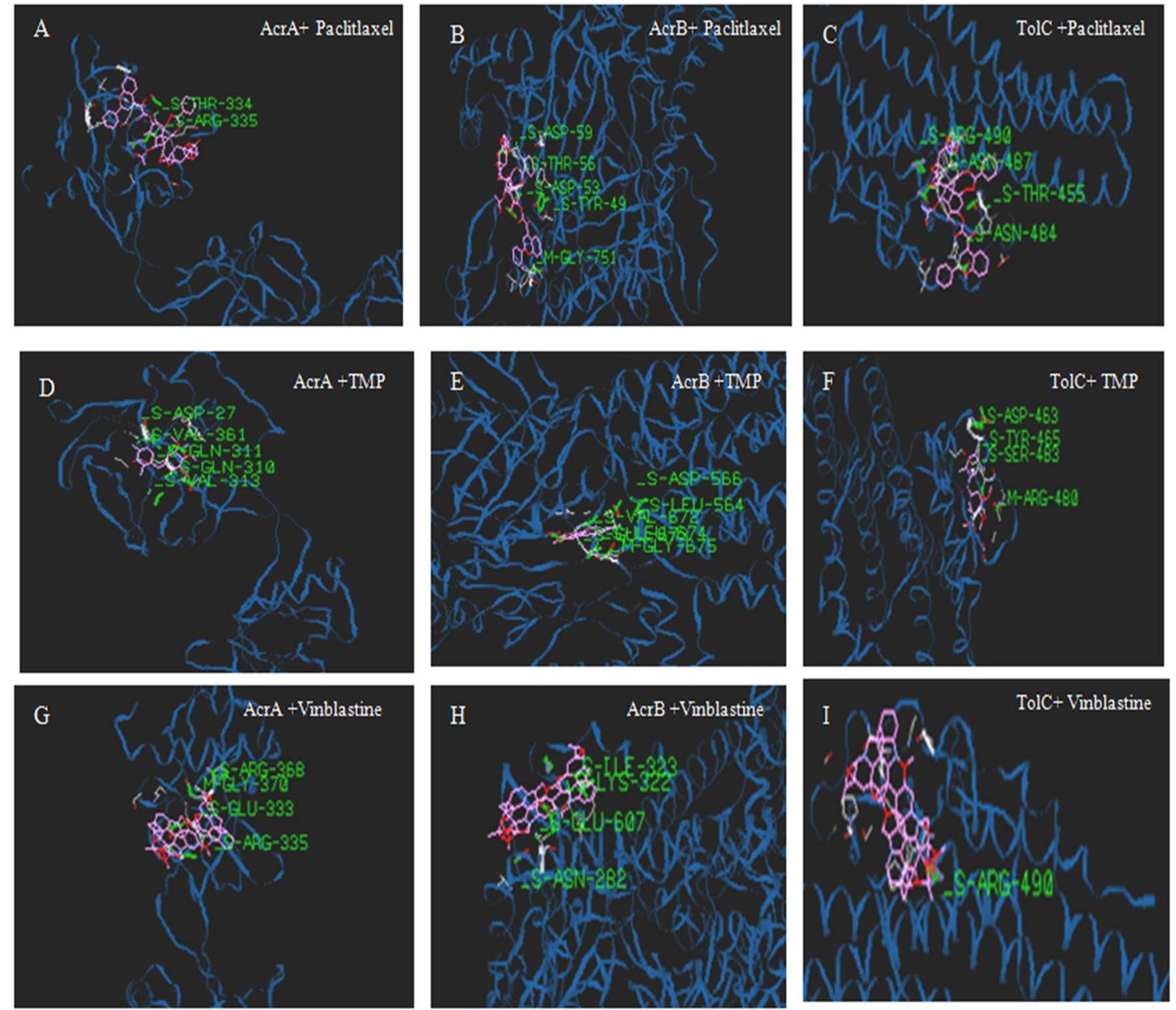

Fig. 4: Docked poses of paclitaxel, vinblastine and trimethoprim with efflux pump subunits AcrA, AcrB and TolC of Salmonella Typhi by iGEMDOCK. Efflux subunits are represented in blue whereas drug ligands are shown in pink. Number of amino acids involved in docked poses has been represented in green

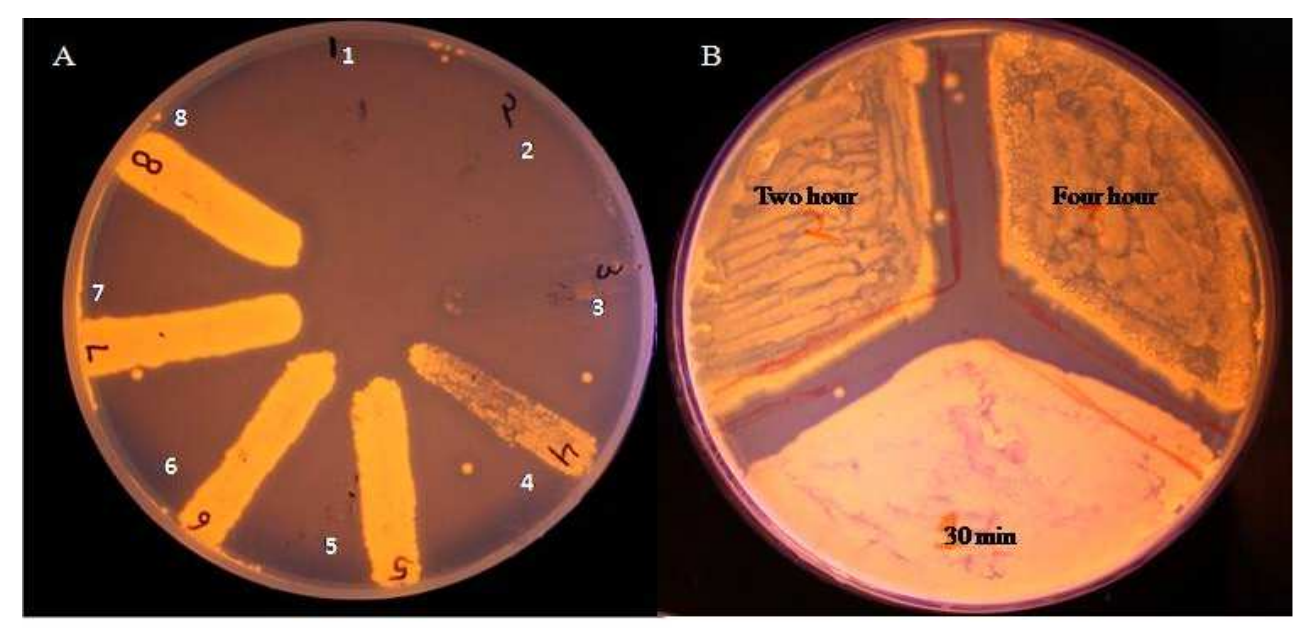

Evaluation of efflux pump activity by EtBr cartwhed assay after drug treatment (paditaxel, vinblastine, trimethopim)

Fig. 5: Evaluation of efflux pump activity of anticancer and antibiotic drug combination EtBr cartwheel assay, Plate A: EtBr containing MHA plate showing the result of induced Salmonella Typhi after its treatment with trimethoprim alone (Spoke 1-4 at 0.5,0.375, 0.25 and 0.125 MIC), vinblastine alone (spoke-5), paclitaxel alone (spoke-6), induced $S$. Typhi (spoke-7), Uninduced $S$. Typhi (spoke-8).

Trimethoprim registered growth of $S$. Typhi culture at only 0.125 MIC (spoke-4), whereas the bacteria failed to grow at $0.5,0.375,0.25$ MIC. Imperceptible change in fluorescence intensities were observed in vinblastine alone, paclitaxel alone, induced and uninduced $S$. Typhi 

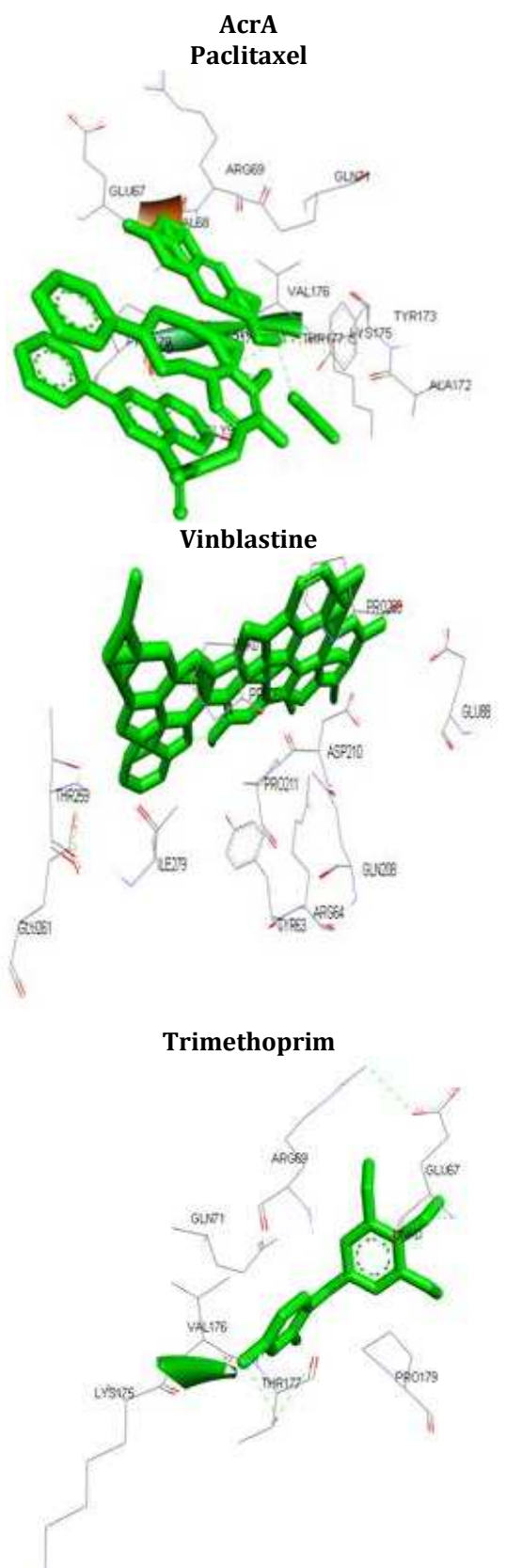

AcrB

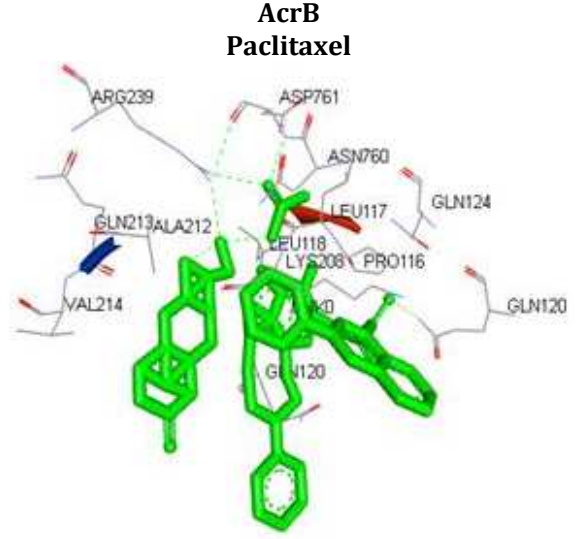

Vinblastine

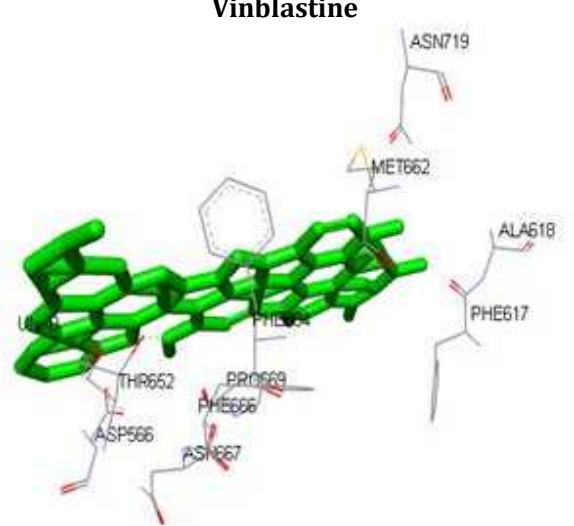

Trimethoprim GLN218

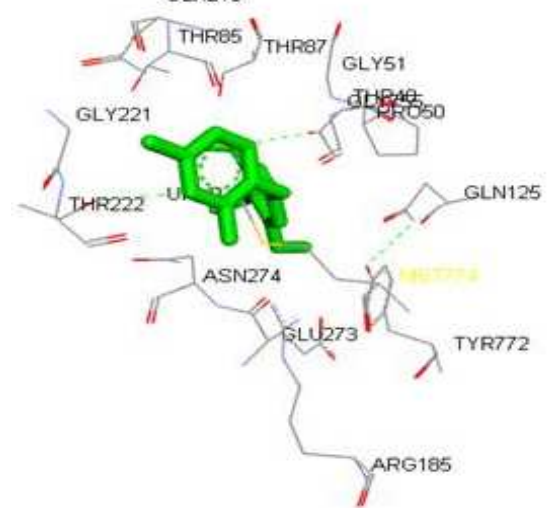

Tolc

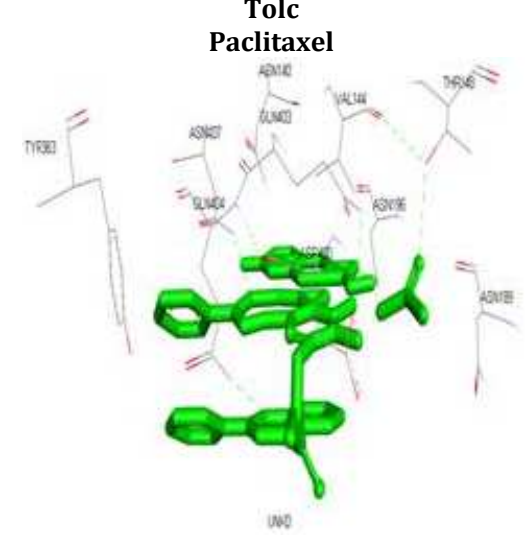

Vinblastine

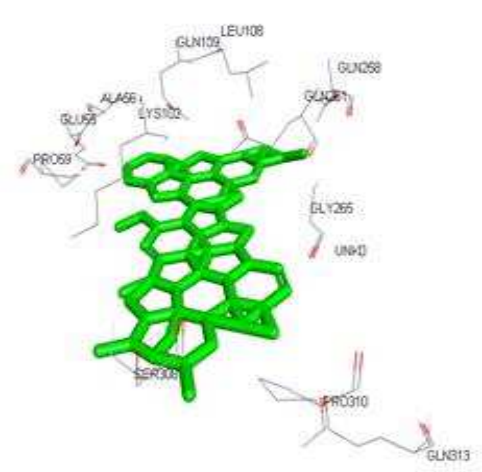

Trimethoprim

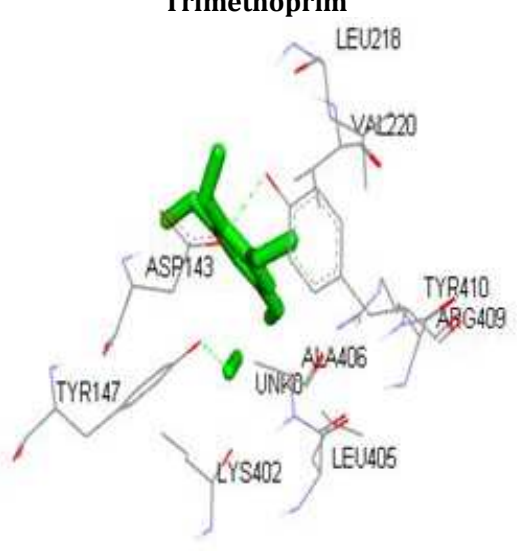

Fig. 6: Docked poses of paclitaxel, vinblastine and trimethoprim with efflux pump subunits AcrA, AcrB and TolC of Salmonella Typhi by Autodock Vina

Typhi over-expressing efflux pumps, as indicated by EtBr cartwheel and semi-automated fluorescent assay

Ethidium bromide cartwheel assay is a simple, instrument free and comparatively easy method to identify several isolates of multidrugresistant pathogen simultaneously on a single agar plate. Ethidium bromide is a common substrate of AcrAB-TolC efflux system, and CFUs of resistant bacteria produce less fluorescence on agar plate under UV light because of high efflux of EtBr from the bacterial cells (fig. 5). A number of efflux pump inhibitors phenylalanine-arginine $\beta$ naphthylamide (PABN) [26-27], 1-1-Naphthylmethyl-piperazine (NMP), pyridopyrimidine one and pyrazolopyridines) exerted their inhibitory activity against bacterial efflux pump at concentration range from $70 \mu \mathrm{g} / \mathrm{ml}$ [28] to $100 \mu \mathrm{g} / \mathrm{ml}$ [29], for example, PAßN registered half maximum activity approximately at $70 \mu \mathrm{g} / \mathrm{ml}$ whereas NMP caused EtBr accumulation at $100 \mu \mathrm{g} / \mathrm{ml}$. An EPI (such as NMP) can also act as antibacterial agent at four-fold $(400 \mu \mathrm{g} / \mathrm{ml})$ concentration to its normal EPI concentration. In the present study, drugs (paclitaxel and vinblastine) failed to observe any effect at concentration ranging from $(10 \mu \mathrm{g} / \mathrm{ml}$ to $160 \mu \mathrm{g} / \mathrm{ml})$ against Salmonella Typhi overexpressing efflux pump on EtBr cartwheel assay suggesting apparent lack of EPI activity, however, insensitive semi-auto fluorescent assay these drug ligand showed significant activity.

Positive control for efflux pump inhibitor (trimethoprim) was unable to produce CFU on EtBr cartwheel assay at different MICs (0.5, 0.375, 0.25 and 0.125 of MIC) because of its bacteriostatic nature.

Ethidium bromide uptake and its expulsion by bacterial efflux pumps have been used previously to ascertain the activity of efflux pump in bacteria. Studies have been reported where MDR bacterial colonies show less fluorescence in the case of the high rate of $\mathrm{EtBr}$ expulsion. The efflux pump inhibitors (EPIs) are reported to produce high fluorescence signals on inhibition of efflux pump activity in EtBr cartwheel assay. Further, efflux pump inhibition can be measured quantitatively by the semi-automated fluorescent assay. 
In the present studies, EPI potential of paclitaxel and vinblastine were determined on indole (at $4 \mathrm{mmol}$ concentration for $4 \mathrm{~h}$ ) induced efflux pump in Salmonella Typhi. Indole triggers the expression of AcrABTolC through RamA in Salmonella species [30], and efflux expression can be detected at $2.5 \mathrm{mg} / \mathrm{ml}$ in MHA medium. Fig. 1 shows the different types of bacteria (E. coli-1, indole treated $S$. Typhi-5, Uninduced $S$. Typhi- 3 and MDR strain of $S$. Typhi) produce differential fluorescence on MHA plate containing $2.5 \mathrm{mg} / \mathrm{ml}$ of EtBr Indole treated $S$. Typhi recorded the least fluorescence confirming overexpression of efflux pump as compared to fluorescence of uninduced $S$. Typhi colonies (fig. 5). Paclitaxel and vinblastine alone reported an imperceptible change in bacterial CFUs fluorescence after thirty minutes of indole-based induction; however, fluorescence diminished after two hours of indole-based induction because of the ample time is required for induction of efflux pumps. Indole is reported to induce all major efflux pumps in Salmonella species.

Paclitaxel and vinblastine produced an indiscernible change in fluorescence at the highest concentration of $160 \mu \mathrm{g} / \mathrm{ml}$ each in $\mathrm{EtBr}$ cartwheel assay rejecting the possibilities for these two drugs as being prospective $S$. Typhi efflux pump inhibitors. Findings of EtBr cartwheel assay were further confirmed by more sensitive semi-automated fluorescent assay showing the result of fluorescence signal obtained in various experimental groups (fig. 2). High fluorescence may signify corresponding inhibition of efflux pump in different groups. Uninduced and trimethoprim (U-TMP-L) treated group recorded highest fluorescence signal as compared to indole-induced trimethoprim (ITMP-L group) treated group, which scored 893 points less fluorescence. We considered minimum $10 \%$ difference in the fluorescent signal as indicative of inhibition. Paclitaxel and vinblastine were tested at a concentration range of $160 \mu \mathrm{g}$ to $10 \mu \mathrm{g}$ for indole-induced groups (as indicated by group P-160 $\mu \mathrm{g}, \mathrm{P}-80 \mu \mathrm{g}, \mathrm{P}-40 \mu \mathrm{g}, \mathrm{P}-20 \mu \mathrm{g}, \mathrm{P}-10 \mu \mathrm{g}$ and V$160 \mu \mathrm{g}, \mathrm{V}-80 \mu \mathrm{g}, \mathrm{V}-40 \mu \mathrm{g}, \mathrm{V}-20 \mu \mathrm{g}, \mathrm{V}-10 \mu \mathrm{g}$ respectively).

Moreover, control groups of uninduced and indole-induced were also included in the experiment. Since the trimethoprim has been shown to be potently antibacterial in EtBr cartwheel, assay its sublethal concentration (U-TMP-LC50 and I-TMP-LC50) was also tested in uninduced and indole-induced bacterial groups. Fluorescence obtained by various concentrations of paclitaxel and vinblastine restricts into a narrow non-significant region as indicated in (fig. 2). When the fluorescence signals of similar concentrations of paclitaxel and vinblastine compared a very low difference of 18 points $(160 \mu \mathrm{g} / \mathrm{ml}), 8$ points $(80 \mu \mathrm{g} / \mathrm{ml}), 41$ points $(40 \mu \mathrm{g} / \mathrm{ml}), 84$ points $(20 \mu \mathrm{g} / \mathrm{ml})$ and 39 points $(10 \mu \mathrm{g} / \mathrm{ml})$ were recorded. Moreover, 628.1 point difference was found in the uninduced and induced group.

Table 1: Data showing interaction analysis of amino acids with different subunits of AcrAB-TolC of $S$. Typhi. H-S signifies hydrogen bond with side chain; H-M signifies hydrogen bond with the main chain. Number of amino acids is shown below its respective amino acid by Auto Dock VINA

\begin{tabular}{|c|c|c|c|}
\hline Anticancer compound & Interaction analysis AcrA & Interaction analysis AcrB & Interaction analysis TolC \\
\hline \multirow[t]{5}{*}{ Vinblastine } & H-S H-S H-S H-M & H-S H-S H-S H-S & $\mathrm{H}-\mathrm{S}$ \\
\hline & GLU ARG ARG GLY & ASN LYS ILE GLU & ARG \\
\hline & 333335368370 & 282322323607 & 490 \\
\hline & $-4.4-10.5-3.5-2.7$ & $-10.6-7-2.5-2.5$ & -15.6 \\
\hline & H-S H-S & H-S H-S H-S H-S H-M & H-S H-S H-S H-S \\
\hline \multirow[t]{4}{*}{ Paclitaxel } & THR ARG & TYR ASP THR ASP GLY & THR ASN ASN ARG \\
\hline & 334335 & 49535659751 & 455484487490 \\
\hline & $-4.9-7$ & $-5-2.5-5-2.5-3.5$ & $-8.1-3.5-3.2-3.5$ \\
\hline & H-S H-S H-S H-S H-S & H-S H-S H-S H-S H-S H-M & H-S H-S H-M H-S \\
\hline \multirow[t]{3}{*}{ Trimethoprim } & ASP GLN GLN VAL VAL & LEU ASP VAL GLU LEU GLY & ASP TYR ARG SER \\
\hline & 27310311313361 & 564566672673674675 & 463465480483 \\
\hline & $-6.9-3.3-8.9-3.5-3.5$ & $-3.5-7.5-3.5-3.5-3-2.5$ & $-3.3-6.5-3.5-8.9$ \\
\hline \multirow[t]{2}{*}{ Anticancer compound } & Interaction analysis & Interaction analysis & Interaction analysis \\
\hline & AcrA & AcrB & TolC \\
\hline \multirow[t]{5}{*}{ Vinblastine } & H-S H-S H-S H-M & H-S H-S H-S H-S & $\mathrm{H}-\mathrm{S}$ \\
\hline & GLU ARG ARG GLY & ASN LYS ILE GLU & ARG \\
\hline & 333335368370 & 282322323607 & 490 \\
\hline & $-4.4-10.5-3.5-2.7$ & $-10.6-7-2.5-2.5$ & -15.6 \\
\hline & H-S H-S & H-S H-S H-S H-S H-M & H-S H-S H-S H-S \\
\hline \multirow[t]{4}{*}{ Paclitaxel } & THR ARG & TYR ASP THR ASP GLY & THR ASN ASN ARG \\
\hline & 334335 & 49535659751 & 455484487490 \\
\hline & $-4.9-7$ & $-5-2.5-5-2.5-3.5$ & $-8.1-3.5-3.2-3.5$ \\
\hline & H-S H-S H-S H-S H-S & H-S H-S H-S H-S H-S H-M & H-S H-S H-M H-S \\
\hline \multirow[t]{3}{*}{ Trimethoprim } & ASP GLN GLN VAL VAL & LEU ASP VAL GLU LEU GLY & ASP TYR ARG SER \\
\hline & 27310311313361 & 564566672673674675 & 463465480483 \\
\hline & $-6.9-3.3-8.9-3.5-3.5$ & $-3.5-7.5-3.5-3.5-3-2.5$ & $-3.3-6.5-3.5-8.9$ \\
\hline
\end{tabular}

Table 2: Tabulated free energy (vdW, Hydrogen bonding energy, and electrostatic energy) of docked poses of paclitaxel, vinblastine and trimethoprim obtained from Autodock Vina

\begin{tabular}{llll}
\hline EPI & Total energy kcal/mol & & \\
\hline Subunits & AcrA & AcrB & \\
Trimethoprim & -5.1 & -7.0 & \\
Vinblastine & -7.6 & -10.2 & -4.8 \\
Paclitaxel & -8.1 & -11.0 & -9.1 \\
\hline
\end{tabular}

Table 3: Data showing interaction analysis of amino acids with different subunits of AcrAB-TolC of $S$. Typhi. Number of amino acids has also been given below each amino acid by Auto Dock Vina

\begin{tabular}{|c|c|c|c|}
\hline Anticancer compound & Interaction analysis AcrA & Interaction analysis AcrB & Interaction analysis TolC \\
\hline \multirow[t]{6}{*}{ Vinblastine } & THR GLU ILE UNK & ASN MET AL. A PHE & PRO GLU ALA LEU \\
\hline & 2592612790 & 719662618617 & 595556108 \\
\hline & PRO PRO ASP PRO & UNK THR PHE PRO & GLN GLNN LYS GLN \\
\hline & 281289210211 & 0652664669 & 109258102261 \\
\hline & GLU TYR ARG GLN & ASN PHE ASP & GLY UNK SER PRO-GLN \\
\hline & 886364208 & 667666566 & 2650308310313 \\
\hline
\end{tabular}




\begin{tabular}{|c|c|c|c|}
\hline \multirow[t]{7}{*}{ Paclitaxel } & GLU GLN VAL ARG & ARG ASP ASN GLN & TYR ASN GLN ASP \\
\hline & 677117669 & 239761760213 & 363407404400 \\
\hline & AL TYR ALA THR & ALA LEU GLN LYS & ASN GLN ASN ASN \\
\hline & 68173172177 & 212117124208 & 140403196196 \\
\hline & LYS & LEU PRO VAL GLN & ASN UNK THR VAL \\
\hline & 175 & 118116214120 & 1890148144 \\
\hline & & UNK 0 & \\
\hline \multirow[t]{6}{*}{ Trimethoprim } & ARG GLN VAL GLU & GLN THR THR GLY & ASP LEU VAL TYR \\
\hline & 697117667 & 2188587221 & 143218220147 \\
\hline & UNK PRO THR LYS & PRO THR THR UNK & ALA TYR LYS UNK \\
\hline & 0179177175 & 050402220 & 4064104020 \\
\hline & & GLN ARG ASN GLU & ARG LEU \\
\hline & & 125185274273 & 409405 \\
\hline
\end{tabular}

Table 4: Tabulated free energy (vdW, Hydrogen bonding energy and electrostatic energy) of docked poses of paclitaxel, vinblastine and trimethoprim obtained from iGEMDOCK

\begin{tabular}{|c|c|c|c|c|c|c|c|c|c|}
\hline \multirow{2}{*}{$\begin{array}{l}\text { EPI } \\
\text { Subunits }\end{array}$} & & & \multicolumn{7}{|c|}{ Total energy kcal/mol } \\
\hline & & & \multicolumn{2}{|c|}{ AcrA } & \multicolumn{2}{|l|}{ AcrB } & \multicolumn{2}{|c|}{ TolC } & \\
\hline Trimethoprim & & & \multicolumn{2}{|l|}{-89.11} & \multicolumn{2}{|l|}{-84.21} & \multicolumn{2}{|c|}{-90.02} & \\
\hline Vinblastine & & & \multicolumn{2}{|l|}{-111.8} & \multicolumn{2}{|l|}{-124.19} & \multicolumn{2}{|c|}{-146.13} & \\
\hline Paclitaxel & & & \multicolumn{2}{|l|}{-120.71} & \multicolumn{2}{|l|}{-135.22} & \multicolumn{2}{|c|}{-127.74} & \\
\hline EPI & vdW & & \multicolumn{4}{|c|}{ H-Bond } & \multicolumn{3}{|c|}{ Electrostatic energy } \\
\hline Subunits & AcrA & AcrB & TolC & AcrA & AcrB & TolC & AcrA & AcrB & TolC \\
\hline Trimethoprim & -63.01 & -60.71 & $-65,42$ & -26.09 & -23.5 & -24.61 & 0 & 0 & 0 \\
\hline Vinblastine & -88.93 & -100.53 & -126.33 & -22.87 & -23.66 & -19.8 & 0 & 0 & 0 \\
\hline Paclitaxel & -105.63 & -112.64 & -104.69 & -15.08 & -22.58 & -23.06 & 0 & 0 & 0 \\
\hline
\end{tabular}

Table 5: Grid box size and grid box parameter of subunits of AcrAB-TolC with drug ligands (paclitaxel, vinblastine, and trimethoprim) generated by Auto dock Vina

\begin{tabular}{llll}
\hline & AcrA-paclitaxel & AcrB-paclitaxel & TolC-paclitaxel \\
\hline Spacing Angstrom & $0.969 \AA$ & $1.000 \AA$ & $1.000 \AA$ \\
Box Size & $126 \times 112 \times 80 \AA$ & $126 \times 115 \times 124 \AA$ & $126 \times 126 \times 126 \AA$ \\
Centre Grid Box & $-6.567 \times-48.68 \times-23.069$ & $-6.42 \times 48.28 \times-23.064$ & $-7.062 \times-46.80 \times-22.06$ \\
& AcrA-vinblastine & AcrB-vinblastine & TolC-vinblastine \\
Spacing Angstrom & $1.000 \AA$ & $1.000 \AA$ & $1.000 \AA$ \\
Box Size & $120 \times 117 \times 126 \AA$ & $126 \times 119 \times 122 \AA$ & $126 \times 112 \times 121 \AA$ \\
Centre Grid Box & $6.557 \times-48.29 \times-22.099$ & $6.566 \times-47.28 \times-23.045$ & $7.082 \times-48.58 \times-21.046$ \\
AcrA-trimethoprim & AcrB-trimethoprim & $1.000 \AA$ & $0.925 \AA$ \\
Bpacing Angstrom & $1.000 \AA$ & $126 \times 126 \times 126 \AA$ & $126 \times 126 \times 126 \AA$ \\
Centre Grid Box & $126 \times 126 \times 126 \AA$ & $6.457 \times-48.29 \times-22.099$ & $6.447 \times-47.29 \times-22.099$ \\
\hline
\end{tabular}

\section{CONCLUSION}

The rationale of the present study came from previous reports proving the effectivity of anticancer drugs in case of drug-resistant pathogens. In the present work, we concluded that paclitaxel and vinblastine cannot be repurposed as antimicrobials against human pathogens in spite of favorable in silico evidence. Computational approach has been employed earlier to obtain leads (drugs like zanamavir, saquinavir, and captopril to name a few) and may shorten the time period involved in the new drug discovery. Moreover, the drug repurposing has opened up new avenues to explore the novel use of pre-existing drugs for other clinical indications. Decades of hard work has resulted in the refined computational tools to predict three-dimensional (3D) protein structures and ligand docking analysis. A number of freely-available online databases have appeared in last few years namely SWISSMODEL, phyre-2, iGEMDOCK and Autodock Vina. These computational tools are being utilized to obtain a lead in resource intensive and lengthy drug discovery process. In present work, our findings suggest that in silico studies may lack in several respects like drug transport, effective drug concentration, and immediate physiological environment for the drug, which are instrumental in mediating the desired drug effects. In silico studies may show binding of structures, but if it always corresponds to inhibition, remains dicey; however the importance of computational tools cannot be ignored by the scientific community in overall drug discovery process.

\section{ACKNOWLEDGMENT}

I would like to express my deep gratitude and thankfulness to Prof. Kamal Ghanshala ji, (President, Graphic Era University, Dehradun, India) for providing financial assistance and moral support for the research work, Prof. LMS Palni (Dean, Department of Biotechnology, GEU) for encouraging scientific exploration in true spirit, Dr. Ashish Thapliyal (HOD, Biotechnology) for departmental facilities. I am highly grateful to Dr. Krishna Mohan Poluri (Assistant Professor IIT, Roorkee, India) for allowing us to access his laboratory facilities to conduct fluorescence related work.

\section{CONFLICT OF INTERESTS}

Declared none

\section{REFERENCES}

1. Rowe B, Ward LR, Threlfall EJ. Multidrug-resistant Salmonella typhi: a worldwide epidemic. Clin Infect Dis 1997;24 Suppl 1:106-9.

2. Piddock LJV. Multidrug resistance efflux pumps not just for resistance. Nat Microbiol 2006;4:629-35.

3. Bhatia R, Narain JP. The growing challenge of antimicrobial resistance in the South-East Asia region are we losing the battle. J Med Res 2010;5:482-6.

4. Sun J, Deng Z, Yan A. Bacterial multidrug efflux pumps mechanism, physiology, and pharmacological exploitations. Biochem Biophys Res Commun 2014;453:254-67. 
5. Ricci V, Blair JM, Piddock LJ. Ram A, which controls the expression of the MDR efflux pump AcrAB-TolC, is regulated by the Lon protease. J Antimicrob Chemother 2014;69:643-50.

6. Lomovskaya 0 , Watkins W. Inhibition of efflux pumps as a novel approach to combat drug resistance in bacteria. J Mol Microbiol Biotechnol 2001;3:225-36.

7. Aygul A. The importance of efflux systems in antibiotic resistance and efflux pump inhibitors in the management of resistance. Mikrobiyol Bul 2015;49:278-91.

8. Oprea TI, Mestres J. Drug repurposing: far beyond new targets for old drugs. AAPS J 2012;14:759-63.

9. Ashburn TT, Thor KB. Drug repositioning: identifying and developing new uses for existing drugs. Nat Rev Drug Discovery 2004;3:673-83.

10. Daisy $\mathrm{P}$, Nivedha RP, Bakiya RH. In silico drug designing approach for biotin protein ligase of mycobacterium tuberculosis. Asian J Pharm Clin Res 2012;103-7.

11. Kalle AM, Rizvi A. Inhibition of bacterial multidrug resistance by celecoxib, a cyclooxygenase- 2 inhibitor. Antimicrob Agents Chemother 2011;55:439-42.

12. Shah Z, Mahbuba R, Turcotte B. The anticancer drug tirapazamine has antimicrobial activity against Escherichia coli, Staphylococcus aureus and clostridium difficile. FEMS Microbiol Lett 2013;347:61-9.

13. Clinical and Laboratory Standards Institute. Performance standards for antimicrobial disk susceptibility testing; approved standard eleventh addition. CLSI document M02-A11 2015. Clinical and Laboratory Standards Institute, Wayne, PA; 2015.

14. Kelly LA, Sternberg MJE. Protein structure prediction on the web case study using phyre server. Nat Protoc 2009;4:363-71.

15. Irwin JJ, Shoichet BK. ZINC-A free database of commercially available compounds for virtual screening. J Chem Inf Model 2005; 45:177-82

16. Dong $\mathrm{Xu}$, Yang $\mathrm{Z}$. Improving the physical realism and structure accuracy of protein models by a two-step atomic-level energy minimization. Biophys J 2011;101:2525-34.

17. Trott 0, Olson AJ. AutoDock vina: improving the speed and accuracy of docking with a new scoring function, efficient optimization and multithreading. J Comput Chem 2010;31:455-61.

18. Yang JM, Chen CC. GEMDOCK: a generic evolutionary method for molecular docking, proteins structure. Funct Bioinf 2004;55:288-4.

19. Martins M, McCusker MP, Viveiros M, Couto I, Fanning S, Pages JM, et al. A simple method for assessment of MDR bacteria for over-expressed efflux pumps. Open Microbiol J 2013;7:72-82.

20. Nikaido E, Giraud E, Baucheron S, Yamasaki S, Wiedemann $\mathrm{A}$, Okamoto $\mathrm{K}$, et al. Effects of indole on drug resistance and virulence of salmonella enterica serovar typhimurium revealed by genome-wide analyses. Gut Pathog 2012;24:213.

21. Viveiros M, Martins A, Paixao L, Rodrigues L, Martins M, Couto I, et al. Demonstration of intrinsic efflux activity of Escherichia coli K-12 AG100 by an automated ethidium bromide method. Int J Antimicrob Agents 2008;31:458-62.

22. Paixao L, Rodrigues L, Couto I, Martins M, Fernandes P. Fluorometric determination of ethidium bromide efflux kinetics in Escherichia coli. J Biol Eng 2009;16:3-18.

23. Murakami S, Nakashima R, Yamashita E, Matsumoto $T$, Yamaguchi A. Crystal structures of a multidrug transporter reveal a functionally rotating mechanism. Nature 2006;443:173-9.

24. Nissink JW, Murray C, Hartshorn M, Verdonk ML, Cole JC, Taylor R, et al. A new test set for validating predictions of protein-ligand interaction. Proteins 2002;49:457-71.

25. Rushdy AA, Mabrouk MI, Abu-Sef FA, Kheiralla ZH, Mohamed Abdel-All S, Saleh NM, et al. Contribution of different mechanisms to the resistance to fluoroquinolones in clinical isolates of Salmonella enterica. Braz J Infect Dis 2013;17:431-7.

26. Eicher T, Cha HJ, Seeger MA, Brandstatter L, El-Delik J, Bohnert JA, et al. Transport of drugs by the multidrug transporter AcrB involves an access and a deep binding pocket that are separated by a switch loop. Proc Natl Acad Sci USA 2012;109:5687-92.

27. Cha HJ, Muller RT, Pos KM. Switch-loop flexibility affects the transport of large drugs by the promiscuous AcrB multidrug efflux transporter. Antimicrob Agents Chemother 2014;58: 4767-72.

28. Lomovskaya O, Bostian KA. Practical applications and feasibility of efflux pump inhibitors in the clinic-a vision for applied use. Biochem Pharmacol 2006;71:910-8.

29. Bohnert JA, Kern WV. Selected aryl piperazines are capable of reversing multidrug resistance in Escherichia coli overexpressing RND efflux pumps. Antimicrob Agents Chemother 2005;49:49849-52.

30. Chowdhury R, Bakshi R, Wang J, Yildirir G, Liu B, Pappas-Brown $\mathrm{V}$, et al. The killing of African trypanosomes by ethidium bromide. PLoS Pathog 2010;6:e1001226.

\section{How to cite this article}

- $\quad$ Pranjali Gupta, Pankaj Gautam, Nishant Rai. Anticancer drugs as prospective efflux pump inhibitors for Salmonella typhi produce conflicting results in in silico and in vitro studies. Int J Pharm Pharm Sci 2016;8(12):244-251. 